\title{
MODELING AND SIMULATION OF A DUAL CHANNEL ACTIVE NOISE CONTROL SYSTEM FOR POWER TRANSFORMER USING FXLMS ALGORITHM
}

\author{
SUNDAY ADETONA ${ }^{* 1}$, RAIFU SALAWU ${ }^{2}$, MORUF ALABI ${ }^{2}$ \\ ${ }^{1}$ Department of Electrical and Electronics Engineering, University of Lagos, Lagos State, \\ Nigeria \\ ${ }^{2}$ Department of Electrical, Electronics and Computer Engineering, Bells University of \\ Technology, Ota, Ogun State, Nigeria
}

\begin{abstract}
Power transformers are known to generate noise, however, active noise emanating from power transformers many at time is unbearable and has been a subject of concern overtime in power engineering. Several studies on it have principally centered on single channel active noise controller (scANC) using fixed step size, which are characterized with the problems such as signal congestion and instability. This study, therefore, employed Filtered-X Least Mean Square (FXLMS) on a dual channel active noise controller (dcANC) using variable step-size. The noise emanating from a 100 MVA, $132 / 33 \mathrm{kV}$ power transformer was captured with the help of a smart phone in flight mode in accordance with IEC 60076-10 standard of $2 \mathrm{~m}$ away from the transformer and $1 \mathrm{~m}$ apart between each measurement. The recorded noises were taken at one-third of the height of the transformer tank, while electromagnetic interference from the phone and others were assumed to be negligible. A dcANC with FXLMS was model mathematically and implemented in Simulink in the MATLAB environment. Noise reduction ratio, loudness unit full scale and mean square error were used as performance metrics. The simulation results obtained showed that the original noise emanating from the power transformer when ANC was not used was found to be $70 \mathrm{~dB}$. When scANC with FXLMS was used, the noise was reduced to $30.55 \mathrm{~dB}$ whereas, when dcANC with the FXLMS was employed it was reduced to $0.19 \mathrm{~dB}$. Also, the MSE value of $-72 \mathrm{~dB}$ was obtained in the proposed dcANC with FXLMS, compared with $-64 \mathrm{~dB}$ obtained from scANC with FXLMS algorithm. The results of the simulation using FXLMS on both scANC and dcANC showed that the performance of the dcANC is comparatively better in term of the stated performance metrics.
\end{abstract}

Keywords: power transformer, noise, active noise controller, LMS algorithm, Simulink

\section{INTRODUCTION}

The main source of electrical power grid (EPG) is the generator, which generates electric power, and then accompanied by transformers for transmission and distribution of the generated power up to the utilization level [1]. For this reason, transformers form the main and the most essential components of EPG. Meanwhile, transformers can either be power transformer for transmission of high voltages such as $330 \mathrm{kV}, 132 \mathrm{kV}, 33 \mathrm{kV}$ etc., or distribution transformers for distribution at low voltages for utilization. Power transformers are usually big and are accompanied with noise [2].

\footnotetext{
* Corresponding author, email sadetona@unilag.edu.ng

(C) 2021 Alma Mater Publishing House
} 
Noise has been proved by WHO as the second source of health hazard to human in the world [3]. And as such, the noise generated from a power transformer installed mostly close to residential area has to be at the level that will not cause harm to the residents there. Hence, it is important to clarify the harmful noise level for human health [4]. Power transformer noise can be core noise, and this is generated by magnetic force in the core due to the vibration of the stator core magnetostriction that causes displacement of the silicon steel sheets [4]. Because the magnetostrictive period is half of the current and the magnetic circuits of the stator core vary in length, the noise mainly includes low harmonic frequency of $100 \mathrm{~Hz}$ when the fundamental frequency of the power transformer is $50 \mathrm{~Hz}$ [5]. The noise level of $50 \mathrm{~dB}$ to $90 \mathrm{~dB}$, resulting from the low harmonic frequency may give rise to human chronic injury and eventually results in neurological diseases [6]. This is known as the dominant source of power transformer noise. Also, it can be load noise caused by current due to load increase, or Fan-Pump noise caused by fan and pump designed as cooling system in the power transformer. Therefore, it is imperative to consider the electrical parameters used and their environmental effects when power transformers are to be designed and operated, because the power transformer used mainly in the urban areas must be at noise level that does not pose threat to human health. Despite this, the existence of noise on the transformer is inevitable.

Mitigation of noise emanating from power transformers can either be done by passive noise controllers (PNC), such as enclosures, barriers and silencers which are traditional approaches employed in the reduction of acoustic noise and unwanted sounds [7]. This technique is strenuous to analyze quantitatively [8], and it is hard to achieve more significant noise decrease because of the environmental restriction and cost [8]. According to [8], this method, although, is relatively successful over a broad frequency range, but can be costly and ineffective for lowfrequency types of acoustic noise. Comparatively, based on the destructive interference of two sound waves, the active noise control (ANC) method which generates a secondary signal with the opposite phase to offset the primary noise can reduce low-frequency noise, such as transformer noise as stated in [6], more effectively, with better controllability, easier installation and lower cost. The transformer ANC system consists of electroacoustic devices and a controller. The ANC system is either single or multiple channels in Feed forward or feedback or Hybrid technique with either Least Mean Square (LMS) [9] or FXLMS [10] or Filtered-U Least Mean Square (FULMS) [11] algorithm, which serves as the control mechanism. The choice of the algorithm determines several crucial aspects of the overall adaptive process, such as existence of sub-optimal solutions, biased optimal solution, and computational complexity.

The need to control the noise emanating from power transformer as it becomes an inevitable requirement of time in order to protect people's health and the environment few researchers have been able to work on using ANC in mitigation of power transformer noise across the world. For instance, a scANC based on LMS algorithm was proposed by [12] and the model was implemented in Simulink in the MATLAB environment using a practical noise from a $100 \mathrm{kVA}$ power transformer. The analysis of the results from the study of [12] shows huge reduction in the power transformer noise. However, the problem with this model is that a fixed step-size is used which limits its functionality, and difficulty in choosing the appropriate step-size. Another problem is the use of LMS algorithm which achieves large noise reduction, but in an event of time delay caused by secondary path, the result may not be accurate and then results in system divergence. Also, noise extraction method based on IEC 60076-10 standard was not established in the study. Another discussion by [6] shows how ANC technique was used to suppress a high-decibel, low frequency power transformer noise using FXLMS adaptive ANC algorithm based on off-line and on-line secondary path modelling. The method proposed by [6] solved the divergence problem due to the existence of the secondary path when time delay occurs. The transformer noise was monitored on-line, and active control system was realized using both software and hardware, which were selected based on the noise feature. The results from the model showed reduction in noise for on-line secondary path is more compared with off-line secondary path algorithm. Also, Genetic algorithm (GA) is used in this proposed model to optimize the convergence coefficient while the effect of the convergence coefficient on the algorithm was analysed using simulation. However, the drawbacks of this model are the technique for the extraction of the noise from the power transformer is absent in the study, and it cannot handle a complex sound pressure.

Having looked into strengths and the weaknesses of the previous methods on power transformer noise reduction, this study aims at complementing the efforts of earlier researchers by using dcANC with FXLMS algorithm and variable step-size to address signal congestion, divergence and time varying problems. It can work for higher sampling rates up to $44.1 \mathrm{kHz}$ and with the noise time of 14.3 seconds, hence, the number of iterations is 631512 . Also, the power transformer noise extraction was based on IEC 60076-10 standard [13]. Therefore, the goal of this study is to model and simulate a dcANC to reduce noise emanating from a 100 MVA practical power transformer maximally, using a FXLMS algorithm. 


\section{METHODOLOGY}

\subsection{Extraction and characterization of noise from power transformer}

The measurement of power transformer noise consisting of sound pressure and intensity can be done using two methods in accordance with IEC 60076-10 standard [13]. The first approach is the use of sound level meter, while for the second method, the measurement is taken from $0.3 \mathrm{~m}$ distance with forced cooling system off, or $2 \mathrm{~m}$ distance with forced cooling system on. Also, the measurement should be taken at half of the tank, if the transformer tank height is less than $2.5 \mathrm{~m}$. But, if the tank height is greater than $2.5 \mathrm{~m}$, then the measurement must be taken at one-third and two-third of the tank height. Measurements are to be separated at $1 \mathrm{~m}$ apart.

Noise from a typical power transformer becomes noticeable when its intensity is higher than the ambient noise. Hence, any sound recorder can be used for the purpose of recording the noise of a power transformer. In this study, the tank height of the power transformer under test is $7.5 \mathrm{~m}$, and a smartphone was used to record the noise of a 100 MVA $132 / 33 \mathrm{kV}$ power transformer at $1 / 3$ the height mentioned, at Ipaja-Ayobo Transmission Station in MPEG-4 AAC file format. Ipaja-Ayobo is a suburb of Lagos in Nigeria. The noises were recorded at six-interval distances of $2 \mathrm{~m}$ away from the transformer and $1 \mathrm{~m}$ apart between each measurement. Meanwhile, the electromagnetic signal interference from the mobile phone, the power line and other noises in the environment were assumed to be negligible.

MATLAB 2019a, 64-bit version 9.6, was used in the implementation of the proposed system. This package can read MP3 and MPEG-4 AAC formats hence, the recorded transformer noise was in a ready-to-use format. The developed MATLAB script then accesses the stored noise on the laptop through a built-in function 'audioread' where the continuous noise signal was discretized, and the sample frequency returned as the output of the function as shown in Figure 1.

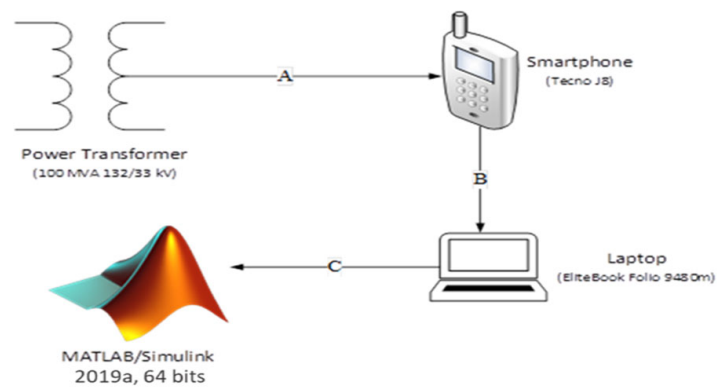

Fig. 1. Experimental setup for the extraction of noise from a power transformer.

\subsection{Development of deANC with FXLMS algorithm}

FXLMS algorithm is a modified version of the LMS algorithm where an extra filter is applied before the adaptive LMS update.

As shown in Figure 2, the noise signal $x(n)$ from the transformer is passed through the primary path $P(z)$, Adaptive filter $W(z)$, and secondary path estimate $\hat{S}(z)$ to obtain the desired signal $d(n), y(n)$ and filtered reference signal $x_{j}^{\prime}(n)$ respectively. In this study, the secondary path estimate, $\hat{S}_{j}(z)$ where $j=1,2$, is modelled as FIR filter with the tap weight length $M ; x_{j}^{\prime}(n)$, which is an input into the LMS update algorithm, and the control filter $W_{j}(z)$ is derived by filtering the reference signal $x_{j}(n)$ through $\hat{S}_{j}(z)$ and is given by:

$$
x_{j}^{\prime}(n)=\hat{s}_{j}^{T}(n) x_{M}(n)
$$

where, $\widehat{s}_{j}{ }^{T}(n)=\left[\hat{s}_{j, 0}(n), \hat{s}_{j, 1}(n), \ldots, \hat{s}_{j, M-1}(n)\right]^{T}$ is the impulse response of $\hat{S}_{j}(z)$ and $x_{M}(n)=[x(n), x(n-$ $1), \ldots, x(n-M+1)]^{T}$ is a $M$ sample reference signal vector. The $M$ sample reference signal vector is obtained as:

$$
x_{M}(n)=e_{M}(n)+y_{M}^{\prime}(n)
$$

where $e_{M}(n)$ is the residual error signal given as: 


$$
\begin{gathered}
e_{j}(n)=d(n)-y_{j}^{\prime}(n) \\
e_{j}(n)= \begin{cases}e_{1}(n), & \text { if } e_{1}(n)<e_{2}(n) \\
e_{2}(n), & \text { otherwise }\end{cases}
\end{gathered}
$$

where $d(n)$ is modelled as the primary disturbance signal, while $y_{j}^{\prime}(n)$ is the cancelling signal which is expressed as:

$$
y^{\prime}(n)=s_{j}(\mathrm{n}) * \mathrm{y}(\mathrm{n})
$$

where $s_{j}(\mathrm{n})$ is the impulse response of the secondary path $S_{j}(\mathrm{z})$, whereas, $y(n)$ is the output signal. $W_{j}(z)$ is modelled as a FIR filter with tap-weight length $L$, therefore, $y(n)$ can be expressed as:

$$
\begin{aligned}
& \mathrm{y}_{j}(\mathrm{n})=w_{j}^{T}(n) x_{L}(n) \\
& \mathrm{y}_{j}(\mathrm{n})=\left\{\begin{array}{lr}
y_{1}(n), & \text { if } y_{1}(n)>y_{2}(n) \\
y_{2}(n), & \text { otherwise }
\end{array}\right.
\end{aligned}
$$

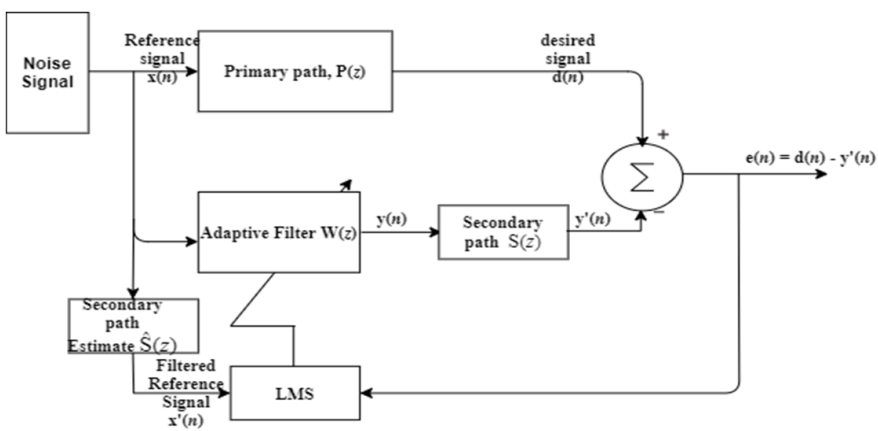

Fig. 2. A block representation of FXLMS algorithm.

In equation $(6), w_{j}^{T}(n)=\left[w_{j, 0}(n), w_{j, 1}(n), \ldots, w_{j, L-1}(n)\right]^{T}$, is the tap-weight vector, and $x_{L}(n)=[x(n), x(n-$ $1), \ldots,(x-L+1)]^{T}$ is a $L$ sample reference signal generator. The noise control filter $W_{j}(z)$ is updated using LMS algorithm as:

$$
w_{j}(n+1)=w_{j}(n)+\mu_{w} e_{j}(n) x_{j}^{\prime}(n)
$$

where $\mu_{w}$ is the step-size for the control filter, $e_{j}(n)$ is the error signal for $W_{j}(z)$ which can be expressed as $e_{j}(n)=$ $d(n)-w_{j}^{T}(n) x_{j}^{\prime}(n)$ and $x_{j}^{\prime}(n)=\left[x_{j}^{\prime}(n), x_{j}^{\prime}(n-1), \ldots, x_{j}^{\prime}(n-L+1)\right]^{T}$ is the filtered reference signal vector.

It is evident from the Figure 2 that the reference signal $x(n)$ is first filtered through the secondary path estimate $\widehat{S}_{j}(z)$. Therefore, the error signal $E_{j}(z)$ can be expressed as:

$$
E_{j}(z)=X(z)\left(P(z)-\hat{S}_{j}(z) W_{j}(z) S_{j}(z)\right)
$$

The error signal $E_{j}(z)$ should be minimized to zero for $W_{j}(z)$ to converge. Hence:

$$
W_{j}(z)=\frac{P(z)}{\hat{S}_{j}(z) S_{j}(z)}
$$

The ratio of the noise output to the desired signal can be given as:

$$
A_{k}=\frac{\sum_{k=1}^{n} e_{k}(n)}{\sum_{k=1}^{n} d_{k}(n)}
$$

In equation (11), $d_{k}(n)$ is the $k^{\text {th }}$ sampled desired audio/noise signal and the ratio in $\mathrm{dB}(\mathrm{A})$ can be expressed as: 


$$
R_{k}=-10 \log _{10}\left(\frac{1}{N} \sum_{k=1}^{N} A_{k}\right)^{2}
$$

where $\left(\frac{1}{N} \sum_{k=1}^{N} A_{k}\right)^{2}$ is the mean of the ratio [14]. The percentage reduction can be expressed as:

$$
R_{\%}=\left(1-10^{R_{k}}\right) \times 100 \%
$$

2.2.1. Mean square error (MSE)

The mean square error (MSE) is a metric indicating how much a system can adapt to a given solution [15]. A small MSE is an indication that the adaptive system has accurately modelled, predicted, adapted and/or converged to a solution for the system [15]. The MSE evaluation of an ANC system is given by:

$$
M S E=E\left[e^{2}(n)\right]
$$

where $e(n)$ and $E[*]$ denotes statistical expectation operator, which is a theoretical function. Equation (14) can be approximately expressed as:

$$
M S E \approx e^{2}(n)
$$

and as such:

$$
M S E=10 \log _{10}\left[e^{2}(n)\right] d B
$$

\section{MODELLING AND SIMULATION}

Figure 3 shows the pseudo code implementation of equations $(1) \div(16)$ whereas its realization in Simulink in the MATLAB environment is presented in Figure 4.

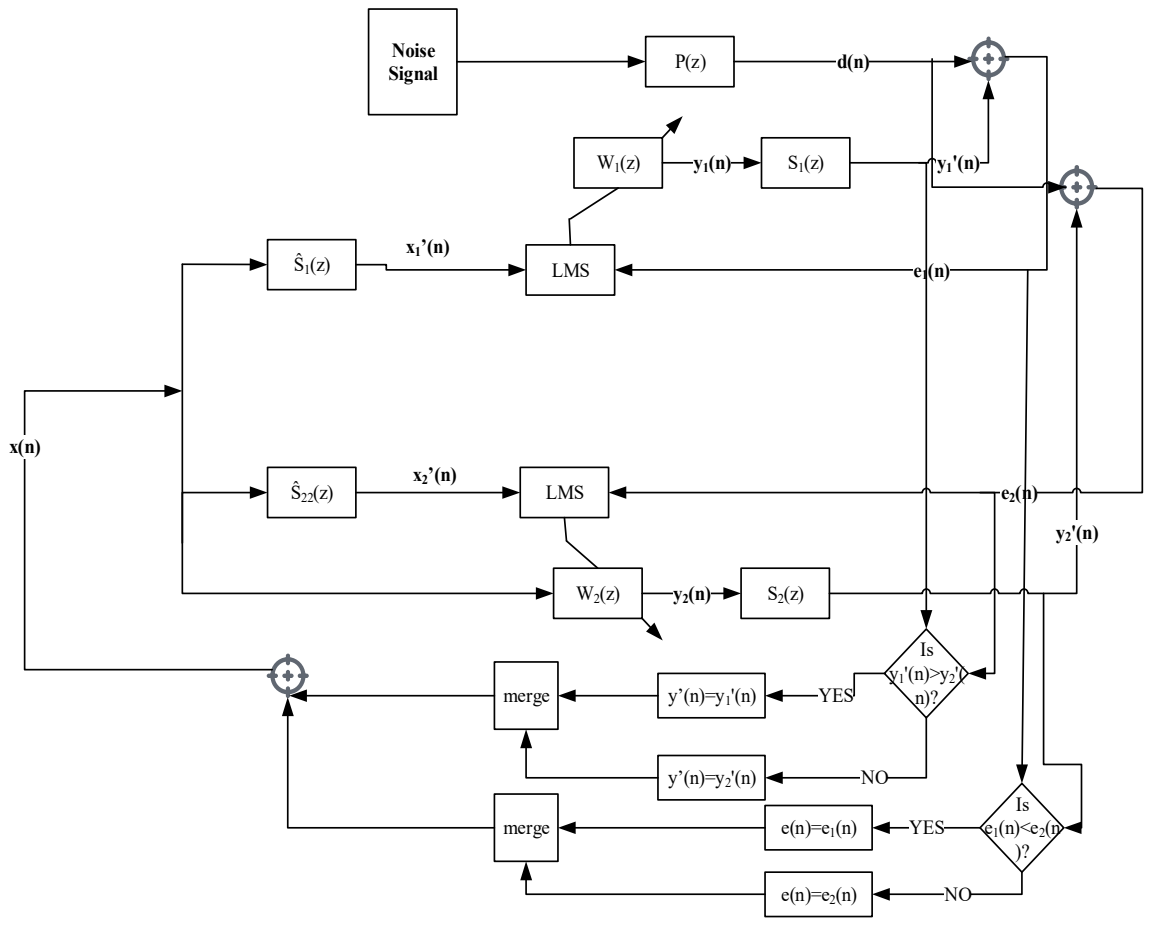

Fig. 3. Pseudo-code of the proposed dcANC with the FXLMS algorithm. 


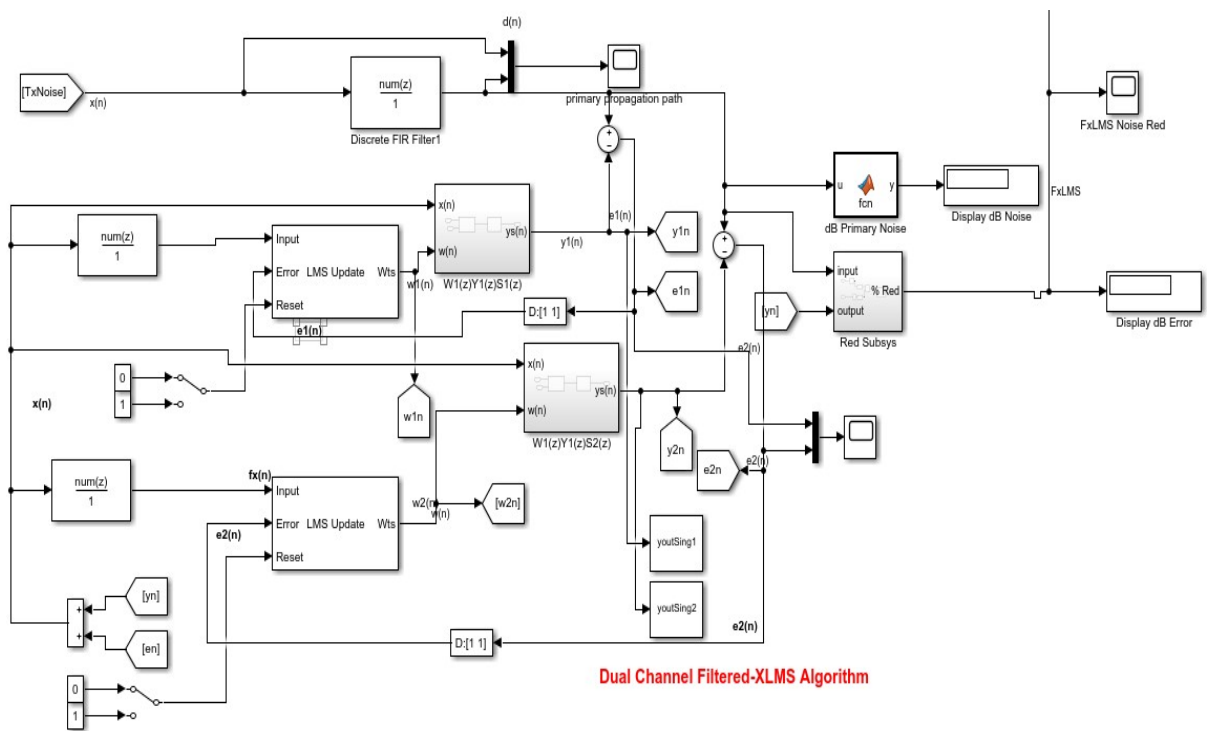

Fig. 4. The Simulink model of the proposed dcANC with the FXLMS algorithm.

\section{SIMULATION RESULTS AND DISCUSSION}

\subsection{Simulation results}

\subsubsection{Extraction and characterization of noise from power transformer}

In this study, six noises were recorded and tested hence the filter length and weight is assumed to be $2^{5}=32$ and the values for step size $\mu_{w}$ are assumed to be in between 0.01 and 0.9 . The power transformer noise is shown in Figure 5 for the first recorded noise taking $1 \mathrm{~m}$ apart according to IEC 60076-10 standard, with loudness of -26.6 Loudness Unit Full Scale (LUFS) as shown in Figure 6.

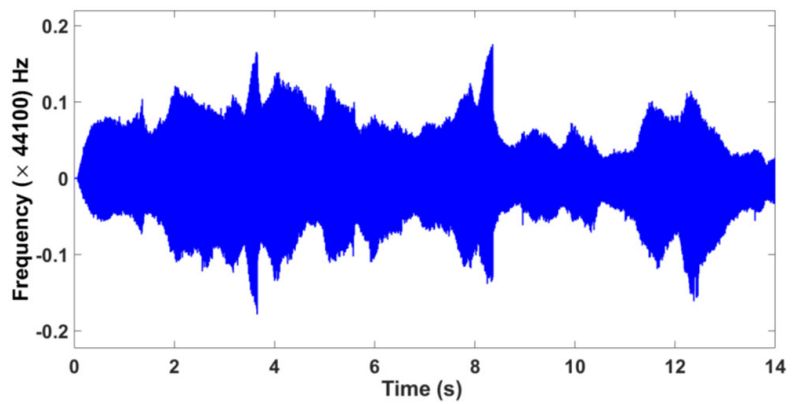

Fig. 5. The waveform of the first recorded original power transformer noise.

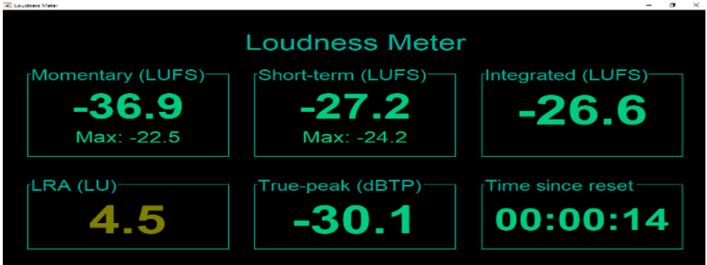

Fig. 6. Loudness meter for the first recorded original noise.

According to European Broadcasting Union R128 standard, this noise loudness level falls within the acceptable limit. However, the number of people around a power transformer is much lower than the number of people seeing a movie, especially when expressed in people-area ratio. Hence, this noise loudness is substantially greater than the ambient noise (relative to the environment) as there is less body to absorb the power transformer noise or generate a noise greater than the power transformer noise. Ambient noise is the background sound level at a given 
location, usually specified as a reference level to study the effect of a noise. In the processing of this noise, one channel is extracted leading to a mono audio. Analysis of the six noises recorded and tested is presented in Table 1 .

Table 1. Recorded Noises outcomes from Loudness Meter (LUFS).

\begin{tabular}{|c|c|c|c|c|c|c|c|}
\hline \multirow{2}{*}{$\begin{array}{c}\text { ANC } \\
\text { Channel }\end{array}$} & $\begin{array}{c}\text { Recorded } \\
\text { Noise }\end{array}$ & $\begin{array}{c}\text { Recorded } \\
\text { Noise }\end{array}$ & $\begin{array}{c}\text { Recorded } \\
\text { Noise }\end{array}$ & $\begin{array}{c}\text { Recorded } \\
\text { Noise }\end{array}$ & $\begin{array}{c}\text { Recorded } \\
\text { Noise }\end{array}$ & $\begin{array}{c}\text { Recorded } \\
\text { Noise }\end{array}$ & \multirow{2}{*}{ Average } \\
\cline { 2 - 7 } Original & -26.6 & -27.2 & -29.0 & -35.8 & -34.9 & -32.8 & -31.1 \\
\hline $\begin{array}{c}\text { FXLMS } \\
\text { (scLMS) }\end{array}$ & -52.2 & -51.1 & -59.9 & -68.9 & -64.5 & -70.5 & -61.2 \\
\hline $\begin{array}{c}\text { FXLMS } \\
\text { (dcLMS) }\end{array}$ & $-\infty$ & $-\infty$ & $-\infty$ & $-\infty$ & $-\infty$ & $-\infty$ & $-\infty$ \\
\hline
\end{tabular}

\subsubsection{The simulation results obtained from scANC with FXLMS Algorithm}

Figure 7 shows the amplitude waveform of the scANC with FXLMS under laid with the original signal for the first recorded noise.

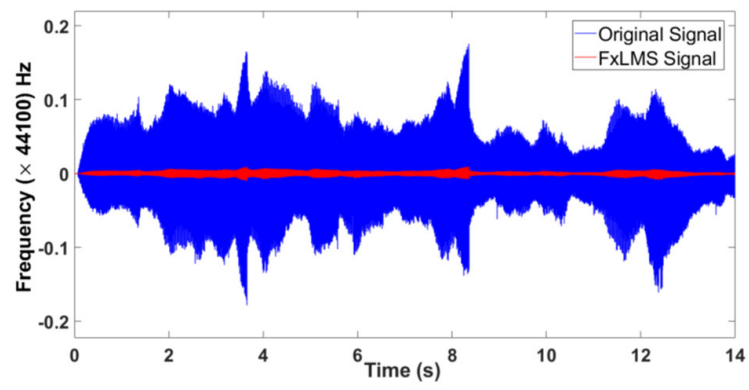

Fig. 7. Original signal overlaid with scANC with FXLMS signal for the first recorded noise.

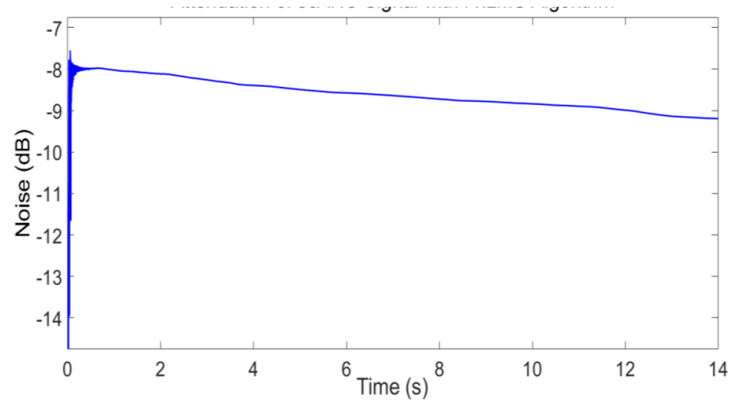

Fig. 8. Attenuation of the obtained signal of scANC with FXLMS algorithm for the first recorded noise.

It can be observed that the amplitude of the signal obtained with scANC with FXLMS algorithm becomes attenuated with time. This is the effect of continue approximation of the filter weights. The attenuation of the noise in $\mathrm{dB}(\mathrm{A})$ is shown in Figure 8 for the first recorded noise. The noise amplitude was reduced to about that of the original first recorded noise.

\subsubsection{The simulation results obtained from dcANC with FXLMS algorithm}

Figure 9 shows the original first recorded noise signal overlaid, by the dcANC with FXLMS obtained signals. It shows that the recorded noise was greatly reduced. The attenuation graph shows that the amplitude of the noise was reduced to approximately zero as shown in Figure 10. The results analysis for the six recorded and tested noises for this study is indicated in Table 2 . 


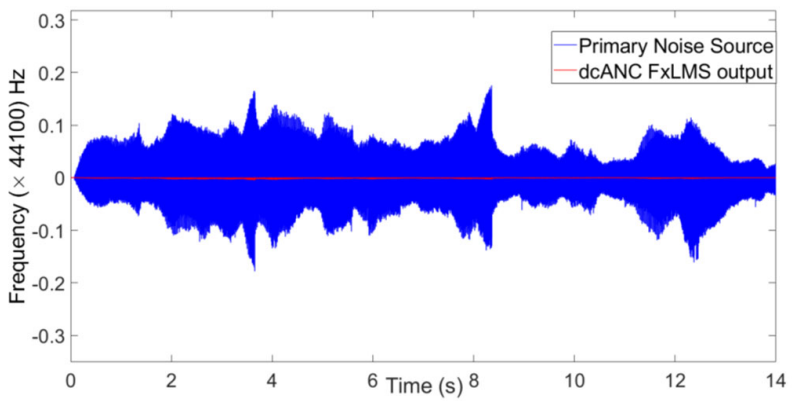

Fig. 9. The first original recorded noise overlaid with dcANC using FXLMS algorithm.

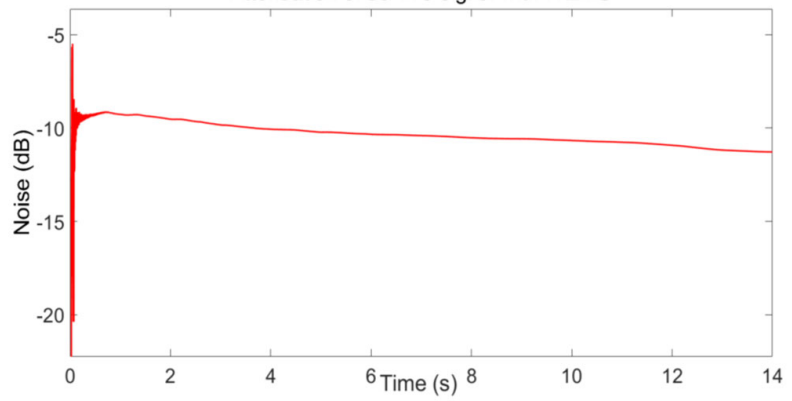

Fig. 10. Attenuation of the first original recorded noise using dcANC with FXLMS algorithm.

Table 2. Simulink model noise $(\mathrm{dB})$ reduction results.

\begin{tabular}{|c|c|c|c|c|c|c|c|}
\hline \multirow{2}{*}{ ANC Channel } & $\begin{array}{c}\text { Recorded } \\
\text { Noise }\end{array}$ & $\begin{array}{c}\text { Recorded } \\
\text { Noise }\end{array}$ & $\begin{array}{c}\text { Recorded } \\
\text { Noise }\end{array}$ & $\begin{array}{c}\text { Recorded } \\
\text { Noise }\end{array}$ & $\begin{array}{c}\text { Recorded } \\
\text { Noise }\end{array}$ & $\begin{array}{c}\text { Recorded } \\
\text { Noise }\end{array}$ & \multirow{2}{*}{ Average } \\
\cline { 2 - 7 } Original & 1 & 2 & 3 & 4 & 5 & 6 & \\
\hline FXLMS (scLMS) & 23.14 & 24.07 & 35.53 & 30.20 & 31.18 & 38.00 & 30.40 \\
\hline FXLMS (dcLMS) & -0.02 & -0.08 & -0.06 & -0.17 & -0.17 & -0.12 & -0.20 \\
\hline
\end{tabular}

The MSE $(\mathrm{dB})$ results of scANC and dcANC for the six recorded and tested noises are presented on the Table 3 to show effective reduction of noise with dcANC system.

Table 3. Model MSE (dB) results.

\begin{tabular}{|c|c|c|c|c|c|c|c|}
\hline \multirow{2}{*}{$\begin{array}{c}\text { ANC } \\
\text { Channel }\end{array}$} & $\begin{array}{c}\text { Recorded } \\
\text { Noise }\end{array}$ & $\begin{array}{c}\text { Recorded } \\
\text { Noise }\end{array}$ & $\begin{array}{c}\text { Recorded } \\
\text { Noise }\end{array}$ & $\begin{array}{c}\text { Recorded } \\
\text { Noise }\end{array}$ & $\begin{array}{c}\text { Recorded } \\
\text { Noise }\end{array}$ & $\begin{array}{c}\text { Recorded } \\
\text { Noise }\end{array}$ & Average \\
\cline { 2 - 7 } $\begin{array}{c}\text { FXLMS } \\
\text { (scLMS) }\end{array}$ & -70.2 & -52.9 & -56.9 & -67.4 & -61.9 & -75.8 & -64.0 \\
\hline $\begin{array}{c}\text { FXLMS } \\
\text { (dcLMS) }\end{array}$ & -79.8 & -47.1 & -69.2 & -72.8 & -79.7 & -85.4 & -72.0 \\
\hline
\end{tabular}

\subsection{Discussion}

Figure 11 shows the original signal, scANC with FXLMS and dcANC with FXLMS signals overlaid on one another. The figure shows that the proposed dcANC with FXLMS algorithm outperforms scANC with FXLMS algorithm. Figure 12 shows the corresponding attenuations. 


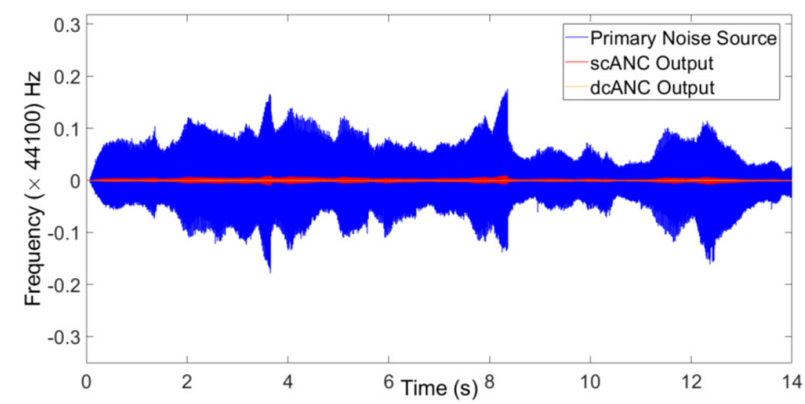

Fig. 11. The first recorded original noise and the resulting signals from scANC and dcANC with FXLMS algorithms overlaid on one another.

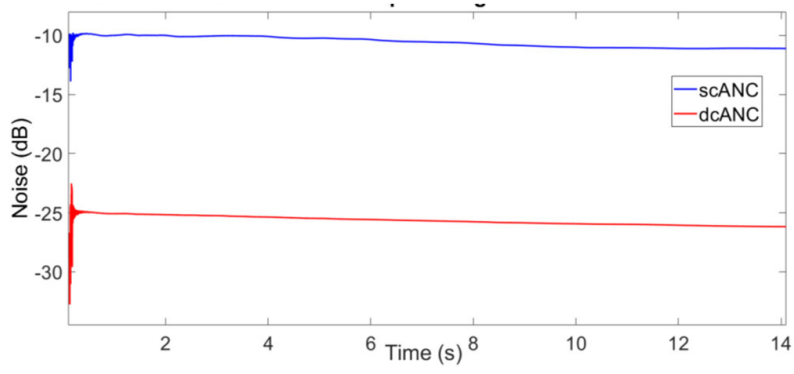

Fig. 12. Attenuation of the first recorded original noise signal using scANC and dcANC with FXLMS algorithms.

Also, analysis of the results of the six (6) tested noises indicated on Table 1 shows that the proposed dcANC with FXLMS performs better than scANC with the same algorithm. The integrated loudness is calculated by breaking the noise signal into 0.4 second segments with 0.3 second overlap. It is a measure of the perception of loudness. The higher the value is, the higher the perceived loudness. Momentary loudness measures the loudness of the past 400 milliseconds. Short term loudness measures the loudness over the past 3 seconds. Loudness range measures the difference between highest recorded short-term loudness and the lowest short-term loudness over the entire duration of the signal. True peak measures inter-sample peaks.

The average value -61.1 LUFS on Table 1 implies that the attenuated noise by scANC with FXLMS algorithm sounds 30 LUFS less loud than the average original noise. The mean value $-\infty$ of the dcANC with FXLMS algorithm on Table 1 implies that the attenuated noise cannot be heard at $44.1 \mathrm{kHz}$ frequency. The attenuation graphs' results presented on Table 2 show approximately $30.5 \mathrm{~dB}$ averagely, for the scANC with FXLMS signal and approximately $-0.19 \mathrm{~dB}$ for the dcANC. This is the ratio of the attenuated noise to the original noise for scANC and dcANC with FXLMS algorithms respectively. This implies that the amplitudes of the original recorded noises were reduced to approximately $30 \mathrm{~dB}$ when scANC is utilized. Equally, the dcANC model reduced the original recorded noises to approximately $0 \mathrm{~dB}$. This explains why the attenuated recorded noises using dcANC with FXLMS algorithm is hardly audible to human ears at $44.1 \mathrm{kHz}$ frequency.

From the simulation results shown on Table 3, it can be observed that the MSEs for dcANC with FXLMS algorithm $(-72.33 \mathrm{~dB})$ is lower than that for scANC $(-64.17 \mathrm{~dB})$. Thus, the former gives better performance of noise reduction. All the results of performance analysis tools used for this study show that dcANC with FXLMS performs better than scANC with FXLMS. This justifies better performance of dcANC at different point of measurements.

\section{CONCLUSIONS}

This study has modeled and simulated a dual channel active noise control system for a 100 MVA power transformer using filtered-x least mean square algorithm. The noise emanating from a typical practical power transformer was obtained and characterized according to IEC 60076-10 standard and neglecting the external interference. The noise and the dcANC with FXLMS algorithm model were implemented in Simulink in the MATLAB environment using DSP, audio and control tools. The results obtained using noise reduction ratio, 
LUFS, and mean square error performance metrics, show that the power transformer noise reduction is greater in the dcANC with FXLMS algorithm when compared with scANC. This study has shown that dcANC with FXLMS using variable step-size is an effective technique for reducing humming noise from a power transformer. Further studies can consider the use of other algorithms that can effectively provide a proper acoustic feedback effect and compared with the result of this research. Also, the use of adaptive IIR filter with recursive least square (IIR-RLS) should also be considered regarding noise reduction in power transformer.

\section{ACKNOWLEDGMENTS}

The authors are pleased to acknowledge the support provided by the Department of Electrical, Electronics and Computer Engineering, Bells University of Technology, Ota, Ogun State, Nigeria.

\section{REFERENCES}

[1] Akrami, A., Doostizadeh, M., Aminifar, F., Power system flexibility: An overview of emergence to evolution, Journal of Modern Power System and Clean Energy, vol. 7, no. 5, 2009, p. 987-1007.

[2] Oyeleye, M.O., Design of $11 / 0.415 \mathrm{kV}$ substation using applicable international codes, Journal of Multidisciplinary Engineering Science Studies, vol. 5, no. 9, 2019, p. 2782-2789.

[3] Sahib, M.A., Streif, S., Design of an active noise controller for reduction of tyre/road interaction noise in environmentally friendly vehicle, IEEE Conference Proceedings, 2017, p. 59-62.

[4] Ming, R.S., Pan, J., Norton, M.P., Wende, S., Huang, H., The sound-field characterisation of a power transformer, Applied Acoustic, vol. 56, no. 4, 1999, p. 257-272.

[5] Negi, R., Singh, P., Shah, G., Causes of noise generation and its mitigation in transformer, International Journal of Advanced Research in Electrical, Electronics and Instrumentation Engineering, vol. 2, no. 5, 2013, p. 1732 1736.

[6] Tong, Z., Jiabi, L., Liang, Z., Li, Z., A new FXLMS algorithm with off-line and on-line secondary-path modelling scheme for active noise control of power transformer, IEEE Transactions on Industrial Electronics, vol. 64, no. 8, 2017, p. 6432-6442.

[7] Kwon, N., Park, M., Lee, H.S., Ahn, J., Shin, M., Construction noise management using active noise control techniques, Journal of Construction Engineering and Management, vol. 142, no. 7, 2016, p. 1-11.

[8] Wen, T., Wen, Y., Zhang, X., Bian, X., Investigating and controlling of power transformer noise, Asia-Pacific Power and Energy Engineering Conference, 2009, p. 1-4.

[9] Kuo, S.M., Morgan, D.R., Active noise control: A tutorial review, Proceedings of the IEEE, vol. 87, no. 6, 1999, p. 943-975.

[10] Rajbala, S.K.G., A review of active noise control system using LMS and FXLMS Algorithm, International Journal of Electronics, Electrical and Computational System, vol. 6, no. 6, 2017, p. 192-196.

[11] Larimore, M.G., Triechler, J.R., Johnson, R.C., An algorithm for adaptive IIR digital filters, IEEE Transaction on Acoustics, Speech, and Signal Processing, vol. 28, no. 4, 1980, p. 428-440.

[12] Batool, M., Yu, C., Shah, M.A., Yuqi, Z., Shiyuan W., Min, Z., Design and simulation using MATLAB/Simulink on active noise control system for power transformer, International Conference on Condition Monitoring and Diagnosis, 2016, p. 465-468.

[13] IEC 60076-10:2001 Standard, Power Transformers part 10: Determination of sound levels.

[14] Gomathi, K., Saravanan, N., Santhiyakumari, N., Variable step size for improving convergence of FXLMS algorithm, Procedia Technology, vol. 25, 2016, p. 420-426.

[15] Mon, A., Aung, T.T., Lwin, C.H., Active noise cancellation in audio signal processing, International Research Journal of Engineering and Technology, vol. 3, no. 11, 2016, p. 21-27. 